\title{
TITLE:
}

\section{First-principles calculations of native defects in tin monoxide}

$\operatorname{AUTHOR}(S):$

Togo, A; Oba, F; Tanaka, I; Tatsumi, K

\section{CITATION:}

Togo, A ...[et al]. First-principles calculations of native defects in tin monoxide. PHYSICAL REVIEW B 2006, 74(19): 195128.

ISSUE DATE:

2006-11

URL:

http://hdl.handle.net/2433/39892

RIGHT:

Copyright 2006 American Physical Society 
PHYSICAL REVIEW B 74, 195128 (2006)

\title{
First-principles calculations of native defects in tin monoxide
}

\author{
A. Togo, F. Oba, and I. Tanaka \\ Department of Materials Science and Engineering, Kyoto University, Sakyo, Kyoto 606-8501, Japan \\ K. Tatsumi \\ Department of Materials, Physics and Energy Engineering, Nagoya University, Chikusa, Nagoya 464-8603, Japan
}

(Received 10 May 2006; revised manuscript received 27 August 2006; published 29 November 2006)

\begin{abstract}
The formation energies and electronic structure of native defects in tin monoxide are investigated by first-principles calculations. Equilibrium defect concentrations, which are obtained using the calculated formation energies and charge neutrality, indicate that the tin vacancy is the dominant defect under oxygen-rich conditions. It forms shallow acceptor states, suggesting that the $p$-type conductivity of tin monoxide originates from the tin vacancy. The equilibrium concentration of the oxygen interstitial is comparable with the tin vacancy at elevated temperatures. However, it is hardly ionized and therefore not expected to contribute to the conductivity. The concentrations of donorlike defects such as the tin interstitial and the oxygen vacancy are low enough not to compensate holes generated by the tin vacancy.
\end{abstract}

DOI: 10.1103/PhysRevB.74.195128

PACS number(s): 61.72.Ji, 61.72.Bb, 71.15.Mb

\section{INTRODUCTION}

Tin oxides have two well-known forms: tin monoxide $(\mathrm{SnO})$ and tin dioxide $\left(\mathrm{SnO}_{2}\right) \cdot \mathrm{SnO}_{2}$ is a prototypical functional material with a wide variety of applications including gas sensors and $n$-type transparent conductive layers, rendering it a target of many researches. In contrast, the physical properties of $\mathrm{SnO}$ have not been well explored. $\mathrm{SnO}$ has a specific electronic structure associated with the presence of divalent tin, $\mathrm{Sn}(\mathrm{II})$, in a layered crystal structure. The unit cell of $\mathrm{SnO}$ is shown in Fig. 1. It is constructed by layered pyramids which are faced and engage each other alternately. The pyramid contains one $\mathrm{Sn}$ atom on the top of the squared four $\mathrm{O}$ atoms. Electrons spread into the open space between the layers, which are often called lone pairs. ${ }^{1,2}$ The presence of a lone pair is expected to generate characteristic physical properties.

Recently, Pan and Fu reported the formation of epitaxial thin films of $\mathrm{SnO}$ by electron-beam deposition. ${ }^{3}$ The films show $p$-type conductivity, and the electrical resistance is $18 \Omega \mathrm{cm}$, in a stark contrast to the $n$-type behavior of $\mathrm{SnO}_{2}$. These results are of interest in view of the fact that most wide-gap oxides with high $p$-type conductivities thus far reported are based on cupper oxide compounds. ${ }^{4}$ The native $p$-type conductivity of $\mathrm{SnO}$ indicates the formation of a substantial amount of native acceptors. Two candidates can be considered for native-acceptor-like defects: the Sn vacancy and the $\mathrm{O}$ interstitial. While cation vacancies form more easily than $\mathrm{O}$ interstitials in most metal oxides with densely packed structures, the layered structure of $\mathrm{SnO}$ with the open spaces surrounded by $\mathrm{Sn}$ (II) may facilitate the formation of $\mathrm{O}$ interstitials. There are several reports on the offstoichiometry of $\mathrm{SnO}, 3,5$ but the defect species relevant to the off-stoichiometry and/or $p$-type conductivity are not well understood. Moreno et al. reported, in their powder crystal study, that $\mathrm{SnO}$ contains a cation deficiency. ${ }^{5} \mathrm{Pan}$ and $\mathrm{Fu}$ also reported the relation between the $p$-type conductivity and the degree of the off-stoichiometry in their epitaxial thin films. ${ }^{3}$
In the present study, we investigate the origin of $p$-type conductivity from the viewpoint of point-defect formation. Employing first-principles calculations with the supercell approach, defect formation energies are obtained as functions of atomic and electronic chemical potentials. We consider the $\mathrm{O}$ interstitial $\left(\mathrm{O}_{i}\right)$, the $\mathrm{O}$ vacancy $\left(V_{\mathrm{O}}\right)$, the $\mathrm{Sn}$ interstitial $\left(\mathrm{Sn}_{i}\right)$, the $\mathrm{Sn}$ vacancy $\left(V_{\mathrm{Sn}}\right)$, and the associations of $\mathrm{O}_{i}$ and $V_{\mathrm{Sn}}$ in relevant charge states, and determine their thermal equilibrium concentrations. The electronic structures of the defects are discussed using one-electronic band structures, local partial density of states, and squared eigenfunctions.

\section{METHOD OF CALCULATION}

\section{A. Defect formation energy and transition energy}

The formation energy of a defect in a charge state $q$ is given $b y^{6}$

$$
\begin{aligned}
\Delta E_{\mathrm{f}}(\text { defect }, q)= & E_{\mathrm{T}}(\text { defect }, q)-E_{\mathrm{T}}(\text { perfect }, q) \\
& +n_{\mathrm{Sn}} \mu_{\mathrm{Sn}}+n_{\mathrm{O}} \mu_{\mathrm{O}}+q\left(\epsilon_{\mathrm{F}}+E_{\mathrm{VBM}}\right),
\end{aligned}
$$

where $E_{\mathrm{T}}($ defect, $q$ ) is the total energy of a supercell with a defect in a charge state $q$ and $E_{\mathrm{T}}$ (perfect, $q$ ) is the total energy of a perfect supercell in a charge state $q . n_{\mathrm{Sn}}$ and $n_{\mathrm{O}}$ are the numbers of $\mathrm{Sn}$ and $\mathrm{O}$ atoms being transferred to (from) atomic reservoir to form a defect, and $\mu_{\mathrm{Sn}}$ and $\mu_{\mathrm{O}}$ are the atomic chemical potentials of $\mathrm{Sn}$ and $\mathrm{O}$, respectively. $\epsilon_{\mathrm{F}}$ is the Fermi level measured from the valence-band maximum (VBM). If $q>0(q<0),|q|$ electrons are transferred from (to) an electron reservoir. The energy at the $\operatorname{VBM}\left(E_{\mathrm{VBM}}\right)$ in a defective supercell is in general different from $E_{\mathrm{VBM}}$ in a perfect supercell when we use finite size supercells with defects under periodic boundary conditions. ${ }^{7}$ Especially, the difference becomes large when the defective model contains highly charged defects. Therefore, it is necessary to line up $E_{\mathrm{VBM}}$ between the perfect and defective supercells. For this purpose, average potentials in the perfect supercell ( $\left.V_{\mathrm{av}}^{\text {perfect }}\right)$ and a bulklike environment which is far from a defect in 


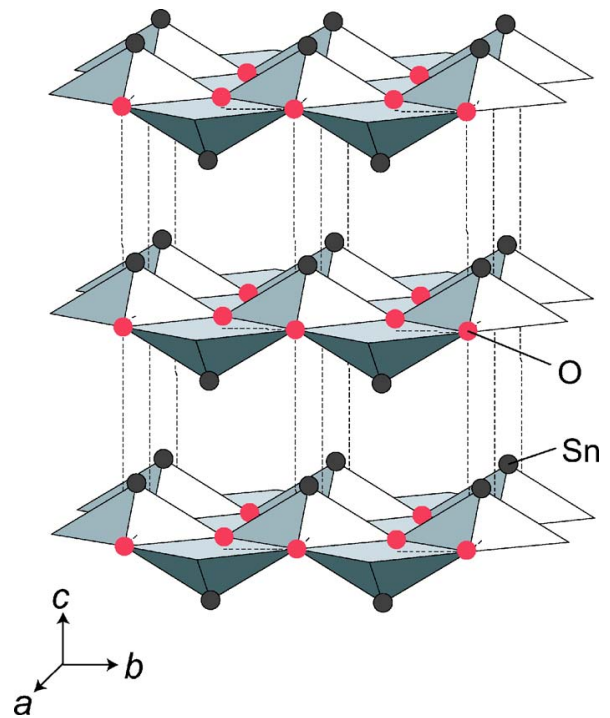

FIG. 1. (Color online) The crystal structure of SnO. Sn and O atoms are depicted by the deep gray circles and the light gray (red) circles, respectively. Four $\mathrm{O}$ atoms and one $\mathrm{Sn}$ atom form a pyramid structure on both sides of each layer alternately. The layers are also stacked alternately.

defective supercells $\left(V_{\mathrm{av}}^{\mathrm{defect}}\right)$ are used as references. ${ }^{8-10} E_{\mathrm{VBM}}$ of a defective supercell is written as

$$
E_{\mathrm{VBM}}=E_{\mathrm{VBM}}^{\text {defect }}=E_{\mathrm{VBM}}^{\text {perfect }}+V_{\mathrm{av}}^{\text {defect }}-V_{\mathrm{av}}^{\text {perfect }},
$$

where $E_{\mathrm{VBM}}^{\text {perfect }}$ was obtained by

$$
E_{\mathrm{VBM}}^{\text {perfect }}=E_{\mathrm{T}}(\text { perfect, } 0)-E_{\mathrm{T}}(\text { perfect, }+1) .
$$

$\mu_{\mathrm{Sn}}$ and $\mu_{\mathrm{O}}$ in Eq. (1) are not independent, but vary between the Sn-rich and O-rich limits under a constraint by the equilibrium condition of SnO. The Sn-rich limit corresponds to the upper limit of $\mu_{\mathrm{Sn}}$ and also the lower limit of $\mu_{\mathrm{O}}$. It is assumed to be determined by solid $\alpha$-Sn-i.e., $\mu_{\mathrm{Sn}}=\mu_{\mathrm{Sn}}^{\alpha \text {-Sn }}$, where $\mu_{\mathrm{Sn}}^{\alpha-\mathrm{Sn}}$ denotes the chemical potential of $\alpha$-Sn. The O-rich limit should be given by the equilibrium condition between $\mathrm{SnO}$ and $\mathrm{SnO}_{2}$. Therefore $\mu_{\mathrm{Sn}}=2 \mu_{\mathrm{SnO}}-\mu_{\mathrm{SnO}_{2}}$ and $\mu_{\mathrm{O}}=\mu_{\mathrm{SnO}_{2}}-\mu_{\mathrm{SnO}}$, where $\mu_{\mathrm{SnO}}$ and $\mu_{\mathrm{SnO}_{2}}$ are defined as the chemical potentials of $\mathrm{SnO}$ and $\mathrm{SnO}_{2}$, respectively. The total energies of $\alpha$-Sn, $\mathrm{SnO}$, and $\mathrm{SnO}_{2}$ obtained from separate calculations are used to determine $\mu_{\mathrm{Sn}}^{\alpha-\mathrm{Sn}}, \mu_{\mathrm{SnO}}$, and $\mu_{\mathrm{SnO}_{2}}$. As a result, $\mu_{\mathrm{Sn}}$ varies by $0.23 \mathrm{eV}$ from the $\mathrm{Sn}$-rich limit to the O-rich limit, which is calculated by $\mu_{\mathrm{Sn}}^{\alpha-\mathrm{Sn}}-2 \mu_{\mathrm{SnO}}-\mu_{\mathrm{SnO}_{2}}$. The formation energies of $\mathrm{SnO}$ and $\mathrm{SnO}_{2}$ are calculated as -2.72 and $-5.21 \mathrm{eV}$, while the values reported by Kılıç and Zunger with the local density approximation (LDA) are -3.21 and $-6.29 \mathrm{eV} .{ }^{11}$ The discrepancies can be attributed to the difference in exchange-correlation potential.

The Fermi level $\epsilon_{\mathrm{F}}$ in Eq. (1) changes its position within the band gap. It is determined by the charge neutrality between concentrations of electrons and holes and charged defects. When we consider the Fermi level as a variable, the defect transition energy $\epsilon\left(q / q^{\prime}\right)$ is described by the Fermi level where the formation energy for a charge state $q$ equals to that for another charge state $q^{\prime}$-i.e.,
TABLE I. The lattice constants of $\mathrm{SnO}$ and the reduced coordinate of the $\mathrm{Sn}$ atom in the $c$ direction.

\begin{tabular}{lccc}
\hline \hline & $a(\AA)$ & $c(\AA)$ & $u$ \\
\hline Calculated & 3.885 & 4.983 & 0.2326 \\
Experimental $^{\mathrm{a}}$ & 3.7986 & 4.8408 & 0.2369 \\
\hline \hline
\end{tabular}

${ }^{\text {aReference } 18 .}$

$$
\epsilon\left(q / q^{\prime}\right)=\left[\Delta E_{\mathrm{f}}^{\mathrm{VBM}}\left(\operatorname{defect} ; q^{\prime}\right)-\Delta E_{\mathrm{f}}^{\mathrm{VBM}}(\operatorname{defect} ; q)\right] /\left(q-q^{\prime}\right),
$$

where $\Delta E_{\mathrm{f}}^{\mathrm{VBM}}($ defect; $q)$ is the defect formation energy for a charge state $q$ when $\epsilon_{\mathrm{F}}$ is at the VBM. Since the band gap energy $E_{\mathrm{g}}$ is represented by the energy difference between the VBM and the conduction-band minimum (CBM), $E_{\mathrm{g}}$ is written as

$$
E_{\mathrm{g}}=E_{\mathrm{CBM}}-E_{\mathrm{VBM}},
$$

where $E_{\mathrm{CBM}}=E_{\mathrm{T}}($ perfect, -1$)-E_{\mathrm{T}}$ (perfect, 0$)$. The band gap of the perfect supercell obtained in this way is $0.29 \mathrm{eV}$, which corresponds to the one-electron energy gap between the $\Gamma$ and $M$ points in the unit cell, $0.37 \mathrm{eV}$, as will be detailed in Sec. III.

\section{B. Computational details}

First-principles calculations were performed in the framework of density functional theory within the generalized gradient approximation ${ }^{12}$ (GGA-PW91) and using plane-wave projector augmented-wave ${ }^{13}$ (PAW) method as implemented in the VASP code. ${ }^{14-16}$ The radial cutoff for the Sn PAW potential is $1.59 \AA$ and that for the O PAW potential $0.80 \AA$. For $\mathrm{Sn}$ atoms, the $5 s$ and $5 p$ electrons were described as valence, whereas for $\mathrm{O}$ the $2 s$ and $2 p$ electrons were treated as valence. The remaining electrons were kept frozen. In order to obtain theoretical structural parameters of $\mathrm{SnO}$, we performed geometry optimization for the unit cell. A planewave energy cutoff of $500 \mathrm{eV}$ was chosen. The Brillouin zone was sampled by a $5 \times 5 \times 4 k$-point mesh generated according to the Monkhorst-Pack (MP) scheme. ${ }^{17}$ The calculated structural parameters are listed in Table I along with the experimental values. The calculated and experimental lattice constants and the reduced coordinate of $\mathrm{Sn}$ atom in the $c$ direction $(u)$ are in agreement within errors of $3 \%$. For $\mathrm{SnO}_{2}$, which contains two $\mathrm{Sn}$ atoms and four $\mathrm{O}$ atoms in the unit cell, and $\alpha-\mathrm{Sn}$, which contains two Sn atoms in the unit cell, the same plane-wave energy cutoff with $\mathrm{SnO}$ was used. The Brillouin zones were sampled by $4 \times 4 \times 5$ and $15 \times 15$ $\times 15$ meshes according to the MP scheme, respectively.

The supercells for defect calculations were constructed from the $48(4 \times 4 \times 3)$ unit cells, which correspond to 192 atoms in the case of perfect crystal. Geometry optimization was performed in fixed lattice constants of $a=b=15.42 \AA$ and $c=14.95 \AA$ which were based on the optimized unit-cell geometry. A plane-wave energy cutoff of $500 \mathrm{eV}$ was chosen and the $k$ point was sampled only at the $\Gamma$ point. The total energies and the forces were converged to less than $10^{-5} \mathrm{eV}$ 


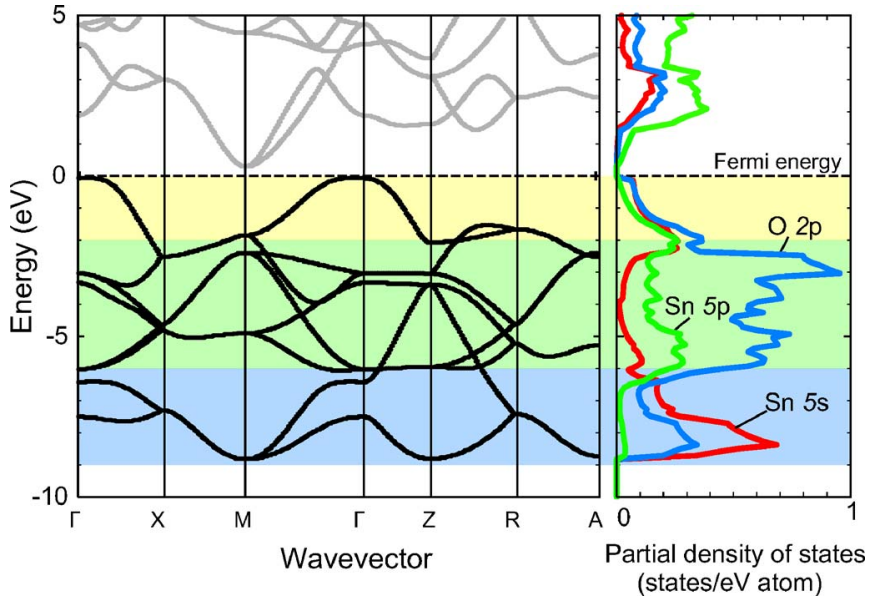

FIG. 2. (Color online) Band structure (left) and PDOS (right) of the unit cell of the SnO perfect crystal. The energy of the highest occupied band at the $\Gamma$ point is set to $0 \mathrm{eV}$. The highest occupied state is located between the $\Gamma$ and $M$ points. The lowest unoccupied state is given at the $M$ point. The background of the band structure and PDOS figures denotes three characteristic energy regions in the valence band.

and $0.05 \mathrm{eV} / \AA$, respectively. For charged defects, a jellium background was employed to neutralize the supercells. The following charge states were considered: -2 to +4 for $\mathrm{Sn}_{i},-2$ to 0 for $V_{\mathrm{Sn}}$ and $\mathrm{O}_{i}$, and 0 to +2 for $V_{\mathrm{O}}$.

\section{RESULTS AND DISCUSSION}

\section{A. Electronic structure of $\mathrm{SnO}$ perfect crystal}

The electronic structure of the perfect crystal is investigated as the basis of the subsequent discussion about the native defects in $\mathrm{SnO}$. Figure 2 shows the calculated band structure and projected density of states (PDOS) of the perfect crystal. The PDOS was obtained by projecting the eigenfunctions onto spherical harmonics around each atom. The states were normalized by a $\mathrm{Sn}$ atom and an $\mathrm{O}$ atom. The integration was made inside each PAW augmentation region whose radius was approximately equivalent to that of each PAW potential. The valence electrons outside the spheres were not considered in the calculations. The Brillouin zone of the unit cell was sampled by a $15 \times 15 \times 12 k$-point mesh according to the MP scheme, and the eigenvalues were interpolated using the improved tetrahedron method. ${ }^{19}$

As shown in Fig. 2 (left), SnO has an indirect band structure. The VBM is located in between the $\Gamma$ and $M$ points and the $\mathrm{CBM}$ is located at the $M$ point. In the valence band, the energy at the $\Gamma$ point is very close to the energy of the highest occupied point. Experimentally, the optical band gap is reported to be $2.5-3.4 \mathrm{eV} .^{20,21}$ In the present calculation, the smallest direct gap is given at the $\Gamma$ point, which is approximately $2.0 \mathrm{eV}$. Although the experimental values scatter in a wide range, a comparison indicates that the present calculation underestimates the gap by $0.5-1.4 \mathrm{eV}$, most likely due to the GGA error.

The PDOS shows that the valence band includes three characters. The lower-energy region $(-9$ to $-6 \mathrm{eV})$, marked
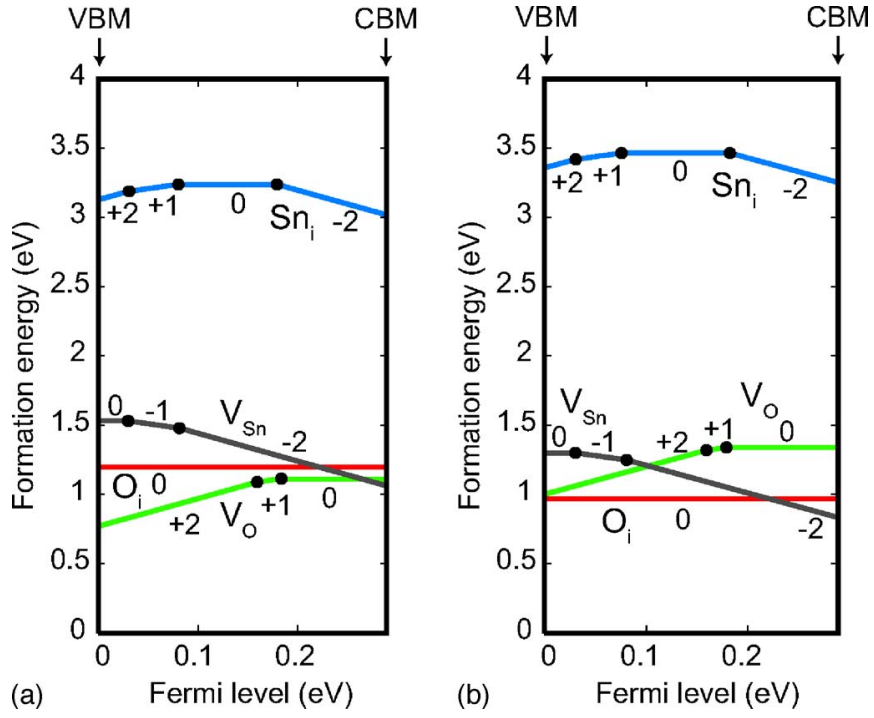

FIG. 3. (Color online) Formation energies as a function of Fermi level. (a) Sn-rich limit. (b) O-rich limit. The numbers alongside the lines designate the predominant charge states of the defects at the Fermi level. The black circles give the defect transition energies in the Fermi level.

by the blue shadow in the figure, is mainly composed of Sn $5 s$ and $\mathrm{O} 2 p$, while $\mathrm{Sn} 5 p$ and $\mathrm{O} 2 p$ mainly constitute the middle region $(-6$ to $-2 \mathrm{eV}$ ) shaded in green color. The higher-energy region $(-2$ to $0 \mathrm{eV})$ indicated by the yellow shadow contains $\mathrm{Sn} 5 s$ and $5 p$ and O $2 p$ components nearly equally, but very near the VBM, the contributions of $\mathrm{Sn} 5 \mathrm{~s}$ and $\mathrm{O} 2 p$ are predominant. In the conduction band, the $\mathrm{O} 2 p$ component is relatively small. The states near the CBM are mainly formed by $\mathrm{Sn} 5 \mathrm{p}$. These features in band structure are in a good contrast to $\mathrm{SnO}_{2}$ which has $\mathrm{O} 2 p$ around the VBM and $\mathrm{Sn} 5 s$ around the CBM as major components. Near the VBM, a large difference in the curvature is recognized between the $\Gamma-X, \Gamma-M$, and $\Gamma-Z$ directions. The effective hole mass in the $Z$ direction near the $\Gamma$ point is smaller than in the $M$ and $X$ directions, suggesting that the $p$-type conductivity of $\mathrm{SnO}$ is anisotropic. Holes may hop easier via lone pairs in the interlayer than in the intralayer region.

\section{B. Defect formation energies}

Defect formation energies as a function of Fermi level are shown in Fig. 3. The Fermi level is measured from the VBM which is set to $0 \mathrm{eV}$. The $\mathrm{CBM}-$ i.e., $E_{\mathrm{g}}$-is calculated at $0.29 \mathrm{eV}$ using the total energy difference as described in Eq. (5). In $\mathrm{SnO}$, two interstitial sites are available in the open spece between layers, which are surrounded by four and five $\mathrm{Sn}$ atoms, respectively. The present calculations indicate that the latter site at the center of the pyramid is energetically more favorable for both $\mathrm{Sn}_{i}$ and $\mathrm{O}_{i}$; therefore, those at this site are shown in the figure.

From the Sn-rich to O-rich limits, the atomic chemical potential varies in a small energy range of $0.23 \mathrm{eV} . V_{\mathrm{Sn}}, V_{\mathrm{O}}$, and $\mathrm{O}_{i}$ show the formation energies of similar magnitude, and the defects with the lowest formation energy change from $V_{\mathrm{O}}$ under $\mathrm{Sn}$-rich conditions to $\mathrm{O}_{i}$ and $V_{\mathrm{Sn}}$ under O-rich 


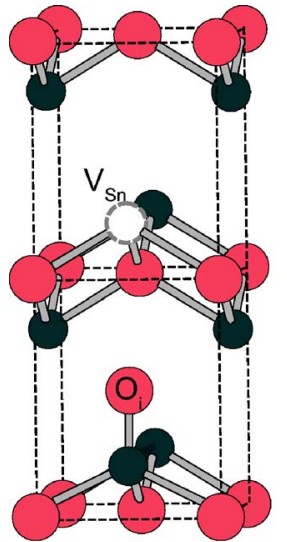

(a)

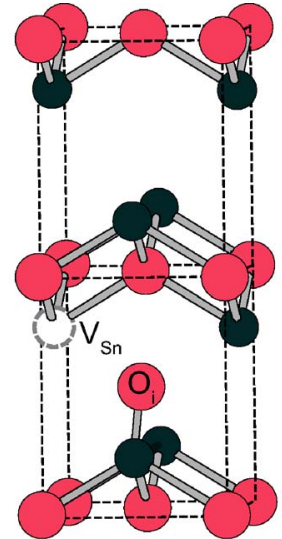

(b)

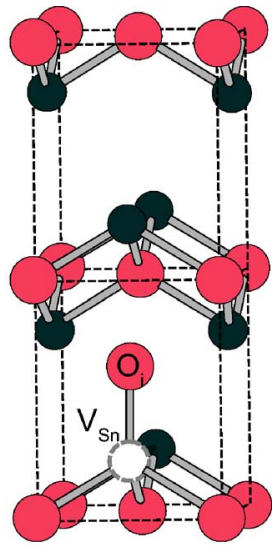

(c)
FIG. 4. (Color online) Three possible models containing $V_{\mathrm{Sn}}$ $-\mathrm{O}_{i}$ pairs. These structures are cut from the supercells. The $\mathrm{Sn}$ and $\mathrm{O}$ atoms are shown as the black and gray (red) circles, respectively. The Sn vacancy position is depicted as the dashed white circle, and the $\mathrm{O}$ interstitial position is shown in the open space between the layers in the bottom unit cell.

conditions. For $V_{\mathrm{Sn}}$ and $V_{\mathrm{O}}$, the energetically most favorable charge states vary with on the Fermi level. The negative- and positive-charge states indicate that these defects are acceptor like and donor like, respectively. The defect transition energies of $V_{\mathrm{Sn}}$ from -2 to -1 and -1 to 0 are estimated at $\sim 0.1 \mathrm{eV}$ from the VBM, and those of $V_{\mathrm{O}}$ from +2 to +1 and +1 to 0 are at $\sim 0.2 \mathrm{eV}$. $\mathrm{O}_{i}$ shows no defect transition level in the calculated band gap. Therefore the existence of $\mathrm{O}$ interstitials is expected not to contribute to the electric conductivity. $\mathrm{Sn}_{i}$ shows both positive- and negative-charge states near the VBM and CBM, respectively. However, the formation energy is considerably high compared with the other defects. $\mathrm{Sn}_{i}$ will be out of consideration in the following discussion since the defect concentration is expected to be much lower than the other defects under thermal equilibrium conditions.

We also investigated associations of $\mathrm{O}_{i}$ and $V_{\mathrm{Sn}}$ using supercells of the same size. Several possible models containing the defect pairs are considered. The structures cut from the supercells after geometry optimization are shown in Fig. 4. The formation energies are summarized in Table II. Models (a) and (b) are similar or slightly higher in formation energy compared with isolated $V_{\mathrm{Sn}}$ and $\mathrm{O}_{i}$, which are obtained independently, and the model (c) is much higher by more than $1 \mathrm{eV}$. The model "Farthest" in Table II contains a Sn vacancy and an $\mathrm{O}$ interstitial which are configured to be farthest away from each other in the supercell. This model shows nearly the same formation energy as the independent one. Thus, the association energy between $V_{\mathrm{Sn}}$ and $\mathrm{O}_{i}$ is negligibly small. In the following discussion, we will focus on isolated defects, especially their behavior at the O-rich limit where acceptorlike defects form most easily.

\section{Corrected defect formation energies}

As mentioned in Sec. III A, the present calculation underestimates the band gap by $0.5-1.4 \mathrm{eV}$. Therefore, the forma-
TABLE II. Formation energies of $V_{\mathrm{Sn}}-\mathrm{O}_{i}$ pairs with charge states 0 and -2 . They are calculated with the Fermi level at the VBM. (a), (b), and (c) correspond to those in Fig. 4. The "Farthest" gives the supercell structure containing the defect pair whose defects are configured to be farthest away from each other in the supercell. The "Independent" gives the sum of the formation energies of $V_{\mathrm{Sn}}^{0}$ and $\mathrm{O}_{i}^{0}$ for the charge state 0 and $V_{\mathrm{Sn}}{ }^{2-}$ and $\mathrm{O}_{i}^{0}$ for the charge state $2-$.

\begin{tabular}{cccccc}
\hline \hline & \multicolumn{5}{c}{ Formation energy (eV) } \\
\cline { 2 - 6 } Charge state & (a) & (b) & (c) & Farthest & Independent \\
\hline 0 & 2.3 & 2.3 & 3.9 & 2.4 & 2.3 \\
-2 & 2.4 & 2.5 & 3.5 & 2.5 & 2.3 \\
\hline
\end{tabular}

tion energies of the defects may include some systematic errors. To correct the errors in the band gap and the formation energies, $E_{\mathrm{CBM}}$ was simply increased to agree with the experimental band gap. The corrected band gap is given by

$$
E_{\mathrm{g}}(\text { corrected })=E_{\mathrm{g}}(\text { calc })+\Delta E_{\mathrm{g}},
$$

where $E_{\mathrm{g}}(\mathrm{calc})$ is the calculated gap for the supercell $(0.29 \mathrm{eV}) . \Delta E_{\mathrm{g}}$ is the difference between the experimental optical band gap and the smallest calculated direct gap $(0.5-1.4 \mathrm{eV})$. Here we employ $\Delta E_{\mathrm{g}}=1.0 \mathrm{eV}$, which results in $E_{\mathrm{g}}($ corrected $)=1.3 \mathrm{eV}$.

We assume that the defect transition energy is shifted upward following $E_{\mathrm{CBM}}$ when the defect state is composed of atomic orbitals similar to those of the CBM. Usually, in such a case, the defect transition energy appears near the CBM since the eigenfunction of the defect transition state hybridizes with the conduction band. Applying this band-gap correction, the defect formation energy is increased by $m \Delta E_{\mathrm{g}}$, where $m$ is the number of electrons at the defect state. ${ }^{22,23}$ Among the considered defects, we applied this formation energy correction only to $V_{\mathrm{O}}$ since the transition energies are close to the CBM and the orbital character of the state is similar to that of the CBM as will be shown in Sec. III E. The corrected formation energies at the O-rich limit are shown in Fig. 5. Compared with the results without the correction as given in Fig. 3, the energy difference between $V_{\mathrm{Sn}}$, $V_{\mathrm{O}}$, and $\mathrm{O}_{i}$ increases with an increase in the Fermi level.

\section{Defect concentrations}

Using the corrected formation energies, concentrations of the defects are determined by charge neutrality under thermodynamic equilibrium condition. The concentration $C$ of a defect can be obtained by

$$
C=N \exp \left(-\Delta H_{\mathrm{f}} / \mathrm{k}_{\mathrm{B}} T\right)
$$

where $N$ is the number of sublattice sites per unit volume. The electron concentration $n$ and the hole concentration $p$ are approximated from the calculated DOS and the statistics distribution functions, as follows: 


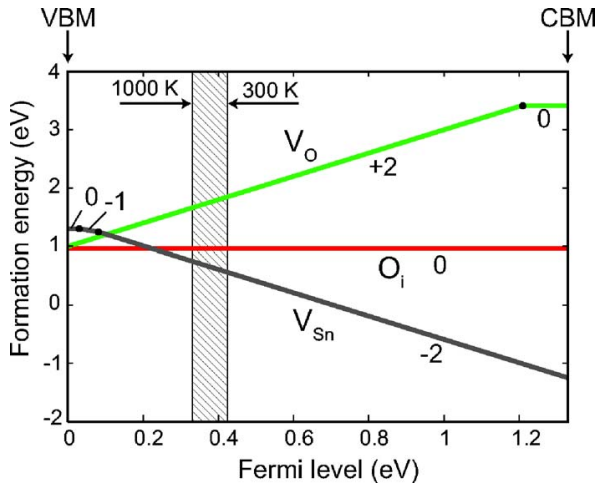

FIG. 5. (Color online) Formation energies as a function of the Fermi level at the O-rich limit after the band-gap correction. The shaded area denotes a variable range of the Fermi level at temperatures between $300 \mathrm{~K}$ and $1000 \mathrm{~K}$ determined from charge neutrality.

$$
n=\int_{E_{\mathrm{CBM}}}^{\infty} D(\epsilon) f_{\mathrm{e}} d \epsilon, \quad p=\int_{-\infty}^{E_{\mathrm{VBM}}} D(\epsilon) f_{\mathrm{h}} d \epsilon,
$$

where $D(\epsilon)$ is the DOS. For $f_{\mathrm{e}}$ and $f_{\mathrm{h}}$, we used the FermiDirac distribution as a function of the Fermi level for electrons and holes, respectively. To substitute the sum of values on a $k$-point mesh for the integral, we sampled the Brillouin zone of the unit cell by a $30 \times 30 \times 30 k$-point mesh at even intervals. Then the equations are rewritten as

$$
\begin{gathered}
n=\sum_{i=\text { unoccupied }} f_{\mathrm{e}}\left(\epsilon_{i}, \epsilon_{\mathrm{F}}\right), \\
p=\sum_{i=\text { occupied }} f_{\mathrm{h}}\left(\epsilon_{i}, \epsilon_{\mathrm{F}}\right),
\end{gathered}
$$

where $\epsilon_{i}$ are the calculated one-electron energies and the in$\operatorname{dex} i$ is shorthand for the occupied bands for $n$ and the unoccupied bands for $p$ and the $k$ points on the $k$-point mesh. The sum is normalized by the density of the $k$ point and the volume of the Brillouin zone. The Fermi level $\epsilon_{\mathrm{F}}$ is considered as a variable here. The charge-neutrality relation among $C, n$, and $p$ is given as

$$
\sum_{\alpha} q_{\alpha} C_{\alpha}-n+p=0
$$

where $q_{\alpha}$ and $C_{\alpha}$ give the charge state and concentration of a defect $\alpha$, respectively. $\alpha$ denotes one of $V_{\mathrm{Sn}}, V_{\mathrm{O}}$, and $\mathrm{O}_{i}$.

In Fig. 6, we show the computed defect concentrations as a function of temperature at the O-rich limit. In the temperature range of $300-1000 \mathrm{~K}$, the major defect is $V_{\mathrm{Sn}}{ }^{2-}$ and the Fermi level is mainly determined by the hole and $V_{\mathrm{Sn}}{ }^{2-}$ concentrations. The electron and $V_{\mathrm{O}}$ concentrations are relatively low and do not affect the Fermi-level position significantly. Increasing the temperature, the Fermi level goes toward the VBM, as shown in the shaded region of Fig. 5, and the $\mathrm{O}_{i}$ concentration becomes comparable with $V_{\mathrm{Sn}}$ in the hightemperature region. However, $\mathrm{O}_{i}$ generates no excess holes or electrons in this Fermi-level range. These results indicate that the $p$-type conductivity of $\mathrm{SnO}$ originates from $V_{\mathrm{Sn}}$.

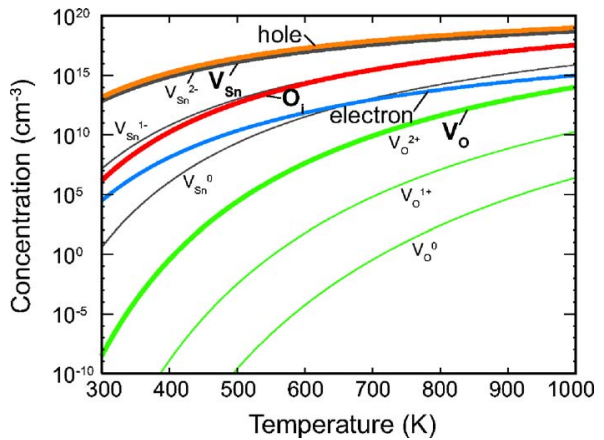

FIG. 6. (Color online) Defect concentrations as a function of temperature at the O-rich limit. $V_{\mathrm{Sn}}$ gives the sum of $V_{\mathrm{Sn}}{ }^{2-}, V_{\mathrm{Sn}}{ }^{-}$, and $V_{\mathrm{Sn}}{ }^{0} \cdot V_{\mathrm{O}}$ gives the sum of $V_{\mathrm{O}}{ }^{2+}, V_{\mathrm{O}}{ }^{+}$, and $V_{\mathrm{O}}{ }^{0}$. The concentrations of $V_{\mathrm{Sn}}$ and $V_{\mathrm{O}}$ are dominated by $V_{\mathrm{Sn}}{ }^{2-}$ and $V_{\mathrm{O}}{ }^{2+}$, respectively.

\section{E. Local electronic structure of defects}

In this subsection, qualitative recognition of local electronic structures in the vicinity of the defects is presented using supercell band structures, squared Kohn-Sham eigenfunctions, and local PDOS (LPDOS). These analyses give intuitive understanding within the one-particle picture. The results are shown in Figs. 7-9, where $0 \mathrm{eV}$ is set to the VBM of the perfect supercell and the reference energy of the defective supercells is aligned with that of the perfect supercell by means of the correction described in Sec. II A. $V_{\mathrm{Sn}}{ }^{2-}$, $V_{\mathrm{O}}{ }^{2+}, \mathrm{O}_{i}{ }^{0}$, and $\mathrm{Sn}_{i}^{2+}$ are selected as representatives of the respective defect species.

The band structures of the perfect and defective supercells are shown in the left side of Figs. 7 and 8. Each band below the band gap is filled with two electrons. The arrows in the supercell band structures indicate eigenvalues for which the cross sections of the squared eigenfunctions are drawn in the right side of Figs. 7 and 8. The contour lines depict an electron density from 0.001 to 0.04 with 0.001 intervals $\left(\AA^{-3}\right)$, where a squared eigenfunction is calculated so that the integral with regard to a state over a supercell volume becomes 2.

The perfect supercell band structure shown in Fig. 7(a) is basically equivalent to that of the unit cell shown in Fig. 2; the reciprocal space of the supercell is folded 4 times for the $a$ and $b$ directions and 3 times for the $c$ direction. The direct band gap at the $\Gamma$ point is narrower than that of the unit cell since the $X$ and $M$ points in the unit cell come to the $\Gamma$ point. In the VBM squared eigenfunction, the electron lone pairs, centered around $\mathrm{Sn}$ atoms, spread into the open space between the layers. O $2 p$ orbitals polarize along the $c$ direction. In the CBM squared eigenfunction, Sn orbitals are delocalized between $\mathrm{Sn}$ atoms in contrast to the VBM squared eigenfunction. The $V_{\mathrm{Sn}}$ supercell band structure shown in Fig. 7(b) is not so much different from that of the perfect supercell. In the $V_{\mathrm{Sn}}$ squared eigenfunction, we can see $\mathrm{O} 2 p$ orbitals next to the $S n$ vacancy rotate by $\sim 45^{\circ}$. In the $V_{\mathrm{O}}$ supercell band structure shown in Fig. 8(a), a defect-induced band splits off from the CBM. The $V_{\mathrm{O}}$ squared eigenfunction shows that this band remains the orbital character of the conduction band. The vacancy site has positive potential and collects electrons which are derived from $\mathrm{Sn} 5 p$ orbitals. In 


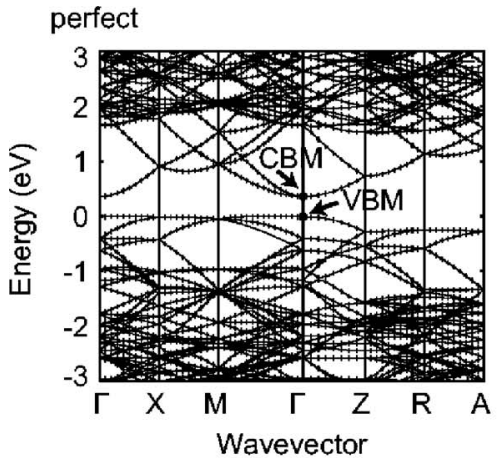

(a)
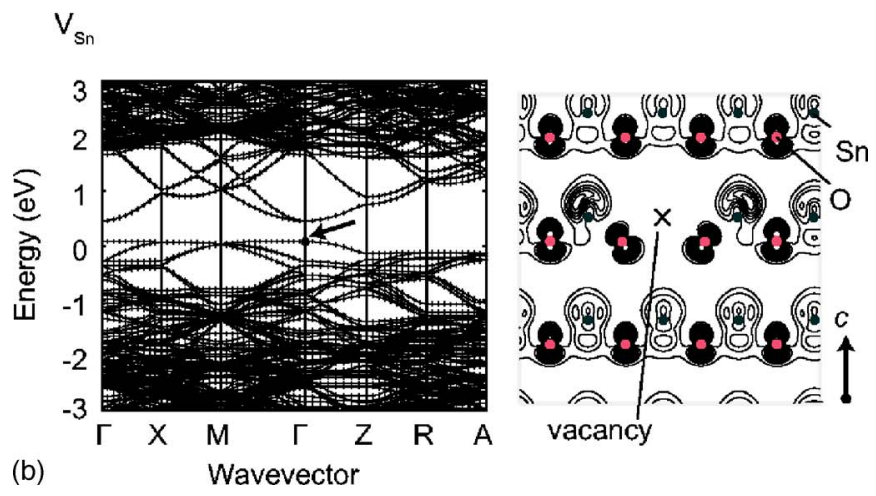

FIG. 7. (Color online) Band structures of the perfect and defective supercells (left) and squared eigenfunctions of the energy levels at the $\Gamma$ point indicated by the arrows in the supercell band structures (right). (a) Perfect crystal. (b) $V_{\mathrm{Sn}}{ }^{2-}$.

the $\mathrm{Sn}_{i}$ supercell band structure shown in Fig. 8(b), two defect-induced bands appear in the band gap and degenerate at the $\Gamma$ point. The $\mathrm{Sn}_{i}$ squared eigenfunction indicates strong electron localization around the $\mathrm{Sn}$ interstitial. Though these energy levels are closer to the VBM than the CBM, they are considered as deep energy levels separated from the conduction band. The $\mathrm{O}_{i}$ supercell band structure shown in Fig. 8(c) is similar to that of the perfect supercell. In the $\mathrm{O}_{i}$ squared eigenfunction, we can identify that the lone pair of the nearest-neighbor $\mathrm{Sn}$ atom, denoted by $\mathrm{Sn}^{*}$, disappears. Further discussion is given below on the basis of the LPDOS. These defective supercell band structures can be classified into two types. $V_{\mathrm{Sn}}$ and $\mathrm{O}_{i}$ have no distinct defect-induced bands inside the band gap. In this case, it is difficult to discuss the defect transition level using the band structure. In the cases of $V_{\mathrm{O}}$ and $\mathrm{Sn}_{i}$, it is relatively easy to have an intuitive understanding of the defect transition level.

The LPDOS of defective supercells were calculated by projecting the eigenfunctions onto spherical harmonics around each focused atom. The integration spheres are equivalent to those written in Sec. III A. All the values obtained were broadened by a Gaussian function $(\sigma=0.1 \mathrm{eV})$.
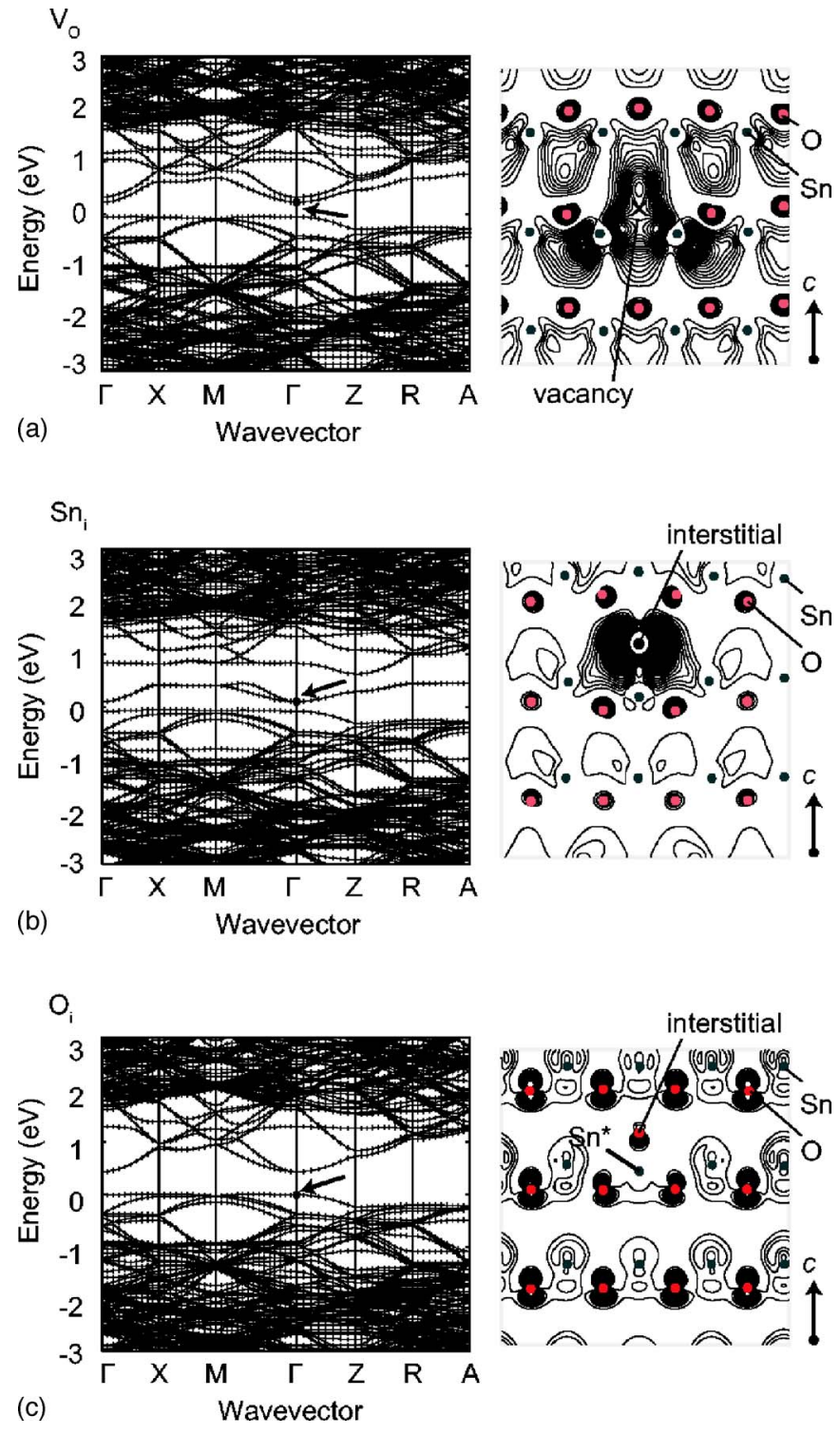

FIG. 8. (Color online) Band structures of the defective supercells (left) and squared eigenfunctions of the energy levels at the $\Gamma$ point indicated by the arrows in the supercell band structures (right). (a) $V_{\mathrm{O}}{ }^{2+}$. (b) $\mathrm{Sn}_{i}{ }^{2+}$. (c) $\mathrm{O}_{i}{ }^{0}$.

The Brillouin zones of the supercells were sampled by a 5 $\times 5 \times 5 k$-point mesh. Figure 9 gives the LPDOS of the interstitial atoms and the nearest-neighbor atoms to the defect sites. The crystal model in the right top shows the defect sites as $V_{\mathrm{Sn}}, V_{\mathrm{O}}, \mathrm{O}_{i}$, and $\mathrm{Sn}_{i}$ and the nearest-neighbor sites as $A, B$, and $C$.

In the LPDOS figures, $\mathrm{Sn} 5 p$ and $\mathrm{O} 2 p$ are divided into $p_{x}+p_{y}$ and $p_{z}$, where $p_{x}$ and $p_{y}$ are represented together since they are symmetrically equivalent. The subscripts $x, y$, and $z$ in $p_{x}, p_{y}$, and $p_{z}$ correspond to $a, b$, and $c$ directions, respectively. The LPDOS of the perfect supercell is basically equivalent to the PDOS shown in Fig. 2 though the LPDOS gives more information about the orientation dependence. The $p_{x}, p_{y}$, and $p_{z}$ states are distributed in separated energy regions clearly. In the energy region between $-2 \mathrm{eV}$ and $0 \mathrm{eV}$, it looks that the $\mathrm{O} 2 p_{z}$ and $\mathrm{Sn} 5 s$ and $5 p_{z}$ make hy- 

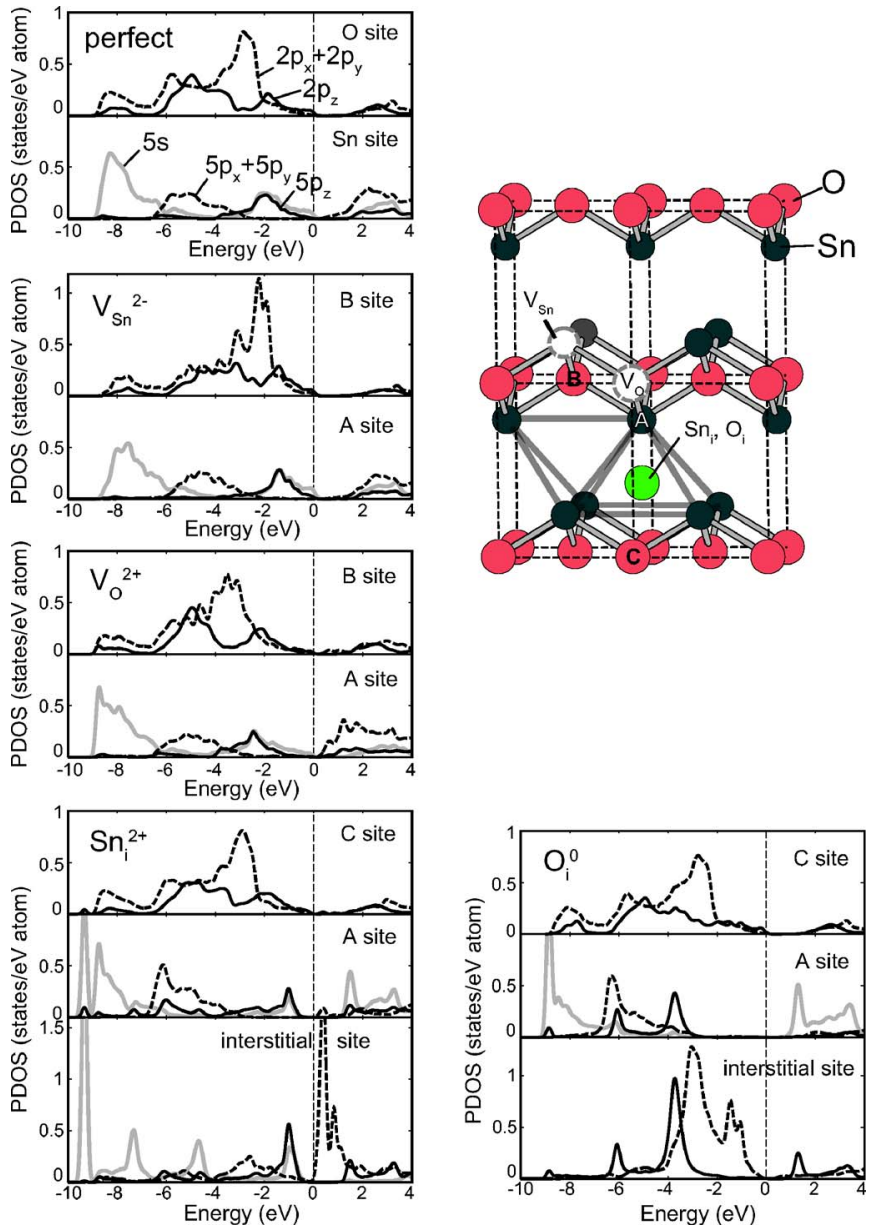

FIG. 9. (Color online) LPDOS of selected atoms in perfect and defective supercells. The positions of atoms considered are shown in right top.

bridized states. In between $-6 \mathrm{eV}$ and $-2 \mathrm{eV}$, the $\mathrm{Sn} 5 p_{x}$ $+5 p_{y}$ and $\mathrm{O} 2 p_{z}$ hybridize. With regard to the perfect $\mathrm{SnO}$ electronic structure, Walsh and Watson gave detailed reports based on first-principles calculations. ${ }^{2}$ Our calculations are consistent to their results. The LPDOS shapes of the $V_{\mathrm{Sn}}$ and $V_{\mathrm{O}}$ supercells are similar to that of the perfect supercell. However, the relative positions of the LPDOS shapes in energy shift upward for $V_{\mathrm{Sn}}$ and downward for $V_{\mathrm{O}}$ since the vacancies distort potentials reaching the vicinal atoms. The states spilling out from the VBM and CBM become acceptorlike and donorlike shallow defect states, respectively. In the LPDOS of the $\mathrm{Sn}_{i}$ supercell, strongly localized $\mathrm{Sn} 5 p_{x}$
$+5 p_{y}$ states appear in the energy region above $0 \mathrm{eV}$. On the contrary, the LPDOS of the nearest-neighbor $\mathrm{Sn}$ and $\mathrm{O}$ atoms are similar to that of the perfect supercell. It is recognized that the interstitial $\mathrm{Sn}$ atom does not significantly disturb the electronic structure of the neighbors and the $\mathrm{Sn}$ interstitial atom leaves itself isolated. In contrast, the insertion of an $\mathrm{O}$ atom into the interstitial site affects drastically the LPDOS shape of the nearest-neighbor $\mathrm{Sn}$ atom at the $A$ site. In between $-3 \mathrm{eV}$ and $0 \mathrm{eV}$, the LPDOS at the interstitial site is relatively high and that at the nearest-neighbor $\mathrm{Sn}$ site has almost disappeared. The nearest-neighbor $\mathrm{O}$ atom at the $\mathrm{C}$ site is not considerably affected. This phenomenon is also confirmed visually by the squared eigenfunction in Fig. 8(c). It appears that the interstitial $\mathrm{O}$ atom absorbs the lone-pair electrons around the $\mathrm{Sn}$ atom at the $A$ site. In other words, the $\mathrm{O}$ and $\mathrm{Sn}$ atoms are ionized to $2-$ and $4+$ in formal charge, respectively.

\section{SUMMARY}

We investigated the formation energies of the native defects in $\mathrm{SnO}$ by first-principles calculations. The equilibrium defect concentrations were estimated from the defect formation energies. The results obtained in this study can be summarized as follows.

(i) The calculations of the defect and carrier concentrations indicate that the $p$-type conductivity of $\mathrm{SnO}$ originates from $\mathrm{Sn}$ vacancies.

(ii) The equilibrium defect concentrations of the Sn interstitial and the $\mathrm{O}$ vacancy are small enough not to compensate holes generated by $\mathrm{Sn}$ vacancies.

(iii) The equilibrium defect concentration of the $\mathrm{O}$ interstitial is comparable with that of the Sn vacancy at elevated temperatures. However, it is hardly ionized and therefore it is not expected to contribute to the conductivity.

(iv) The interstitial $\mathrm{O}$ atom absorbs the lone-pair electrons of the nearest-neighbor Sn atom. In consequence, it is expected that the $\mathrm{Sn}$ atom becomes tetravalent in formal charge.

\section{ACKNOWLEDGMENTS}

The authors would like to thank X. Q. Pan for helpful discussions. This work was supported by Grant-in-Aids for Scientific Research (B) and Young Scientists (B) and the 21st Century COE program, all from the Ministry of Education, Culture, Sports, Science and Technology of Japan.
${ }^{1}$ G. W. Watson, J. Chem. Phys. 114, 758 (2001).

${ }^{2}$ A. Walsh and G. W. Watson, Phys. Rev. B 70, 235114 (2004).

${ }^{3}$ X. Q. Pan and L. Fu, J. Electroceram. 7, 35 (2001).

${ }^{4}$ H. Kawazoe, M. Yasukawa, H. Hyodo, M. Kurita, H. Yanagi, and H. Hosono, Nature (London) 389, 939 (1997).

${ }^{5}$ M. S. Moreno, A. Varela, and L. C. Otero-Díaz, Phys. Rev. B 56, 5186 (1997).
${ }^{6}$ S. B. Zhang and J. E. Northrup, Phys. Rev. Lett. 67, 2339 (1991).

${ }^{7}$ G. Makov and M. C. Payne, Phys. Rev. B 51, 4014 (1995).

${ }^{8}$ T. Mattila and A. Zunger, Phys. Rev. B 58, 1367 (1998).

${ }^{9}$ S. Pöykkö, M. J. Puska, and R. M. Nieminen, Phys. Rev. B 53, 3813 (1996).

${ }^{10}$ C. G. Van de Walle and R. M. Martin, Phys. Rev. B 35, 8154 (1987). 
${ }^{11}$ Ç. Kiliç and A. Zunger, Phys. Rev. Lett. 88, 095501 (2002).

${ }^{12}$ J. P. Perdew, J. A. Chevary, S. H. Vosko, K. A. Jackson, M. R. Pederson, D. J. Singh, and C. Fiolhais, Phys. Rev. B 46, 6671 (1992).

${ }^{13}$ P. E. Blöchl, Phys. Rev. B 50, 17953 (1994).

${ }^{14}$ G. Kresse, J. Non-Cryst. Solids 193, 222 (1995).

${ }^{15}$ G. Kresse and J. Furthmüller, Comput. Mater. Sci. 6, 15 (1996).

${ }^{16}$ G. Kresse and D. Joubert, Phys. Rev. B 59, 1758 (1999).

${ }^{17}$ H. Monkhorst and J. Pack, Phys. Rev. B 13, 5188 (1976).

${ }^{18}$ F. Izumi, J. Solid State Chem. 38, 381 (1981).
${ }^{19}$ P. E. Blöchl, O. Jepsen, and O. K. Andersen, Phys. Rev. B 49, 16223 (1994).

${ }^{20}$ J. Geurts, S. Rau, W. Richter, and F. Schmitte, Thin Solid Films 121, 217 (1984).

${ }^{21}$ R. Sivaramasubramaniam, M. Muhamad, and S. Radhakrishna, Phys. Status Solidi A 136, 215 (1993).

${ }^{22}$ S. B. Zhang, S. H. Wei, A. Zunger, and H. Katayama-Yoshida, Phys. Rev. B 57, 9642 (1998).

${ }^{23}$ K. Matsunaga, T. Tanaka, T. Yamamoto, and Y. Ikuhara, Phys. Rev. B 68, 085110 (2003). 